\title{
Estrategias de comprensión del lenguaje. El desarrollo de la capacidad metalingüística
}

\section{Susana López Ornat}

Universidad Complutense de Madrid.

\section{INTRODUCCION}

Se denomina metacognitivo al proceso que se caracteriza por el conocimiento y control voluntario de los propios procesos evolutivos (Marchesi, 1982). El control se realiza sobre la información, la toma de decisión y la comprobación de los resultados (Wilensky, 198I).

En lo que respecta al lenguaje, Jacobson denominó a esta capacidad «función lingüística», definiéndola como aquella función del lenguaje que le permite referirse a sí mismo. En términos psicolingüísticos esta habilidad recibe el nombre de habilidad metalingüística, y es la que permite utilizar el lenguaje para conocer el lenguaje. En nuestro caso, la habilidad metalingüistica ha sido estudiada solamente en lo que concierne a la comprensión del lenguaje oral. La habilidad de comprensión metalingüística del lenguaje oral equivale a la capacidad (que hemos denominado «nivel formal») que tenga el niño para comprender el lenguaje escuchado guiándose por el lenguaje en sí y no por otros tipos de informaciones no contenidas en el mensaje lingüístico.
El niño, de todos es sabido, va accediendo al modo metalingüístico de relación con el lenguaje (lenguaje-como-objeto) a partir de sus juegos de "prácticas" con el lenguaje.

En el trabajo realizado por $\mathrm{R}$. Wein (1970) sobre los soliloquios de Anthony (dos años) en la cuna, Jacobson comenta que el lenguaje interno vocalizado de Anthony muestra una intima conexión entre las funciones poética y metalingüística, lo que no es característico de los adultos; comenta asimismo que muchas de las grabaciones de los soliloquios son casi idénticas a los ejercicios de gramática y léxico que se encuentran en los libros editados para aprender uno mismo una lengua extranjera.

De hecho, si supiésemos más sobre la adquisición de la habilidad metalingüística, podríamos abordar mejor las técnicas de enseñanza de una segunda lengua. En la práctica, una de las técnicas que se utilizan para este aprendizaje es metalingüística: la comprensión del lenguaje (en nuestro caso, la segunda lengua) mediante el lenguaje (el texto escrito en la lengua nativa del que aprende). Seria, pues, inte- 
resante, saber cuándo y cómo introducir el lenguaje nativo para ayudar al aprendizaje de la segunda lengua.

Esto es un aspecto concreto de la aplicabilidad general de trabajos de este tipo. El rango general de aplicación afecta a cuándo y cómo el lenguaje es un vehículo suficiente para la comprensión, planificación y operativización de cualquier género de problema lingüistico (como en el ejemplo anterior) o no-lingüístico (aprendizaje de conceptos físicos por ejemplo).

Siguiendo con la definición de habilidad metalingüística, P. A. de Villiers y J. G. de Villiers (1980) plantean que alos niños aprenden el lenguaje para la comunicación..., pero más adelante el lenguaje se convierte en un objeto, del cual se toma conciencia... Esto puede demostrarse con respecto a los diferentes niveles del lenguaje: sonido, vocablos y gramátican.

Nuestros trabajos han planteado este proceso de toma de conciencia del lenguaje, explicitado en la comprensión, tanto para los vocablos como para la gramática. (Vocablos: López Ornat, I 977; gramática: López Ornat, 1974, 1975 y 1981.) En todos ellos se ha tratado de centrar el objeto de estudio en cómo se produce ese proceso, cuáles son, si se quiere, los pasos que el niño tiene que dar hasta conseguir esa toma de conciencia, esas habilidades metalingüisticas. A este nivel «final» de desarrollo lo denominamos «nivel formal». El nivel formal se concreta en las habilidades que le permiten al niño comprender de modo metalingüístico el lenguaje.

Para Piaget (1976), la toma de conciencia «... es un proceso que consiste, desde el principio, en una conceptualización propiamente dicha, o sea, en un paso de la asimilación práctica (asimilación del objeto a un esquema) a una asimilación por conceptos» (pág. 259). "Pero en este proceso el niño pasa por diferentes grados de conciencia» (pág. 263), en función de diferentes grados de integración. Estos equivaldrian a lo que hemos definido como «niveles». Nuestros «niveles» no son "estadios», sino conjuntos de habilidades para comprender el lenguaje que se diferencian por el grado de relación que el niño necesita establecer con el contexto de la verbalización para comprenderla.

Nuestra tarea se centra en la descripción de las conductas de comprensión del lenguaje oral que caracteriza a los niños de cada nivel.

Metodológicamente, pues, el trabajo consiste en someter a verificación experimental una predicción sobre las conductas concretas de los niños en cada nivel, identificando los efectos de la edad por procesos de maduración (Baltes, Reese, Nesselroade, 1981).

\section{HIPOTESIS}

\subsection{Hipótesis primera}

Esta hipótesis propone como modelo un sistema de cuatro niveles como marco "estable» para la ontogénesis de la comprensión del lenguaje oral. De tener utilidad científica, ésta sería la de proporcionar conceptos generalizables al estudio de la adquisición del lenguaje. De hecho, y con algunas excepciones (K. Wexler y $\mathrm{P}$. W. Culicover, I980), la variedad y complejidad de formas que hay que aprender a comprender parece haber llevado a la psicolingüística a constreñir sus estudios a la adquisición de una peculiar estructura, proporcionando, como decíamos antes, escasez en cuanto al sentido evolutivo de esos fenómenos concretos.

Parafraseando a Lila Gleitman (198 I), diriamos que nuestra hipótesis parece del tipo "huevo-renacuajo-rana" porque no explica el paso de un nivel a otro por aprendizaje, sino por una suerte de «metamorfosis». Esta apariencia se sustenta en que los niveles están definidos por el modo de relación que el niño necesita establecer con el contexto de la verbalización para comprenderla. Es decir: algunos niños, los más pequeños, no comprenden aunque se les proporcionen todas las claves contextuales (intra y extralingüísticas) de la verbalización estudiada (nivel inferior); otros sí comprenden en esas condiciones, pero dejan de comprender si se les retiran las claves contextuales (nivel 
espontáneo). El grupo de edad inmediatamente superior puede comprender esa misma verbalización sin claves contextuales (CC), presentes "aquí y ahora» y directamente perceptibles, pero deja de comprender cuando no puede elicitar esas CC internamente. Es decir, apoya su comprensión en una referencia interna concreta que le permite aplicar a la comprensión de «esta verbalización» la estrategia que aplicó antes para «aquella otra verbalización» y que le resultó exitosa. Sin embargo, la evidencia experimental demuestra que, al retirar a estos niños toda posibilidad referencial, pierden de nuevo la comprensión de las verbalizaciones.

Este modo de relación (referencial) con el contexto (CC) define el nivel referencial de la comprensión.

El cuarto y último nivel lo constituyen los niños (mayores) que pueden comprender la verbalización de un modo acontextuado (el experimentador no les proporciona claves referenciales ni claves de contexto). Este es el nivel en el que aparece la comprensión metalingüística, la comprensión de la verbalización como tal (nivel formal). ¿Cuál es aquí la relación del niño con el contexto de emisión de la frase? Lo que la hipótesis propone es que no es necesario para el niño apoyarse en ningún contexto presente, consciente, interno ni externo para comprender. Si este contexto es inconsciente, o está subsumido en la maduración neurológica, no es nuestro objeto de estudio.

Los niveles de adquisición de la comprensión se definen, pues, por el modo de relación consciente $y$ actual que el niño necesita establecer con el contexto de la verbalización para comprenderla.

Se podrá objetar, con Reynolds y Ortony (1980), que el contexto está siempre presente, y que incluso los adultos fallan la comprensión cuando se les retira. Ello invalidaría el criterio evolutivo que hemos expuesto. Nuestro trabajo no afecta al contexto de la emisión (que obviamente siempre existe), sino al contexto de la verbalización (referente de la verbalización).

Por otro lado, los sujetos de Ortony et al. (1978) trabajaron sobre la comprensión de metáforas. Distinguieron entre «lenguaje natural», "símil» y «metáfora». Una verbalización metafórica puede no ser comprensible (aun para los adultos) en ausencia del contexto. Pero el contexto de una metáfora es más amplio, incluye otra verbalización, aquella con la que se la compara. De no incluirse, deja de ser metáfora para pasar a "adivinanza». Una objeción así dejaria de tener relevancia para nuestro trabajo.

Pero volvamos al tema que nos ocupaba. Una razón, ya explicada, de la apariencia de metamorfosis, es que aquí no estamos considerando ni el desarrollo neurológico, ni el nivel no-consciente de lo aprendido (factores ambos que podrian dotar de apariencia continua a los niveles que hipotetizamos), sino que sólo estamos haciendo referencia a la evolución cognitiva del niño. En este punto pensamos, como plantean Oleron (1972) y Cromer (1978), que, si el desarrollo cognitivo lo permite, el niño accederá a los problemas de comprensión del lenguaje, pero que ese desarrollo cognitivo es condición necesaria, pero no suficiente para la aparición de una habilidad metalingüística, ya que ésta depende también de la escala de complejidad del lenguaje como tal (complejidad sintáctica y semántica en nuestro caso).

En conclusión, con respecto a nuestra primera hipótesis, ésta no prejuzga ni explica los factores que pueden ser responsables del paso de un nivel al siguiente. La apariencia de "metamorfosis" es sólo una apariencia derivada de nuestra constricción del objeto de estudio a la comprensión del lenguaje oral.

Habría otra pregunta que hacer: ¿de dónde «esos» niveles? Como hemos mencionado antes, esos cuatro niveles propuestos son coherentes con las teorias vigentes en cuanto al desarrollo cognitivo. La evolución de lo cognitivo corre de lo concreto a lo abstracto, para todos los procesos estudiados. Más exactamente: de la necesidad de referentes concretos a la posibilidad de formalización.

Sin embargo, los niños que se sitúan en las fases de transición entre los dos niveles, concreto y abstracto, han sido 
frecuentemente entendidos como niños que oscilan entre ambas posiciones, que saben que el «esquema» anterior es inválido pero aún no pueden operativizar el esquema superior (G. Mugny, A. N. Perret-Clermont, W. Doise, 1978). El comportamiento de estos niños es calificado como de «intermedio" y sus ejecuciones como oscilatorias, entre conductas que caracterizan el nivel anterior y el inmediatamente superior.

Nuestras tres primeras investigaciones fueron realizadas de acuerdo a la hipótesis de dos niveles evolutivos (López Ornat, I975a, b, c, 1977), un nivel concreto denominado "espontáneo" y un nivel formal denominado "elaborado», ello tanto para la adquisición de la comprensión como para la producción del lenguaje oral.

Un estudio posterior (López Ornat, 198I) realizado con 45 niños sobre el mismo problema que el estudio actual, mostró entre ambos niveles un tercer «nivel intermedio", compuesto por niños de edades entre seis y ocho años, situados en una «tierra de nadie» entre el nivel espontáneo (de cinco a seis años) y el nivel "elaborado" (formal) (mayores de ocho años). Su conducta consistía en la emisión de respuestas incoherentes: unas de nivel espontáneo y otras de nivel formal.

Estos datos sobre niños «intermedios» nos llevaron a pensar en la posibilidad alternativa de que se tratase de un tipo de conducta especifica, autónoma y no «intermedia». Aparecería, según la hipótesis, como intermedia debido a no baber sido prevista en el diseño de investigación.

Es éste un problema frecuente en psicolingüística evolutiva. Asi, Eve C. Clark (1973), en un trabajo sobre la adquisición de reglas de interpretación semántica, propone tres estadios. En el primero, los niños se apoyan en estrategias no lingüísticas, y en un conocimiento semántico parcial de las palabras; en segundo lugar propone un estadio de transición, y luego un tercer estadio en que los niños se apoyan en un conocimiento semántico total de las palabras.

Como deciamos al principio, definimos la capacidad de comprensión metalingüís- tica como la capacidad para comprender el lenguaje-como-objeto, expresada por ejemplo en juicios de gramaticalidad, en «juegos» con el lenguaje, y en general en las actividades cuyo objeto y cuyo instrumento es el lenguaje.

Evidentemente, el niño de comprensión "espontánea» está aún muy lejos de la comprensión metalingüística. Su comprensión de las palabras' se apoya todavía sobre la comprensión del contexto intra y extralingüistico, sobre claves gestuales y situacionales, por ejemplo, y sobre el contenido general del mensaje. Por tanto, como muestran los datos obtenidos, este mismo niño pierde la comprensión cuando se le retiran de la situación presente las claves contextuales. El niño de nivel formal puede entender sin presencia de estas claves, y emitir juicios sobre la corrección o incorrección de la verbalización dada. Los niños «intermedios» podrian ser los que ya no necesitasen una contextuación externa, pero sí una interna. Niños que tuviesen que concretar «internamente», a modo de una abstracción concretada ( $\mathrm{J}$. Piaget, 1980). Algo parecido a ponerse un ejemplo concreto para, refiriéndolo al problema planteado, solucionar éste. Un estadio, pues referencial. Si el niño puede ponerse un ejemplo interno concreto relativo a una situación en la que si comprendió la verbalización, aplicará esa misma solución a un problema de comprensión que en abstracto le es irresoluble.

\subsection{Hipótesis segunda}

Otro aspecto de la problemática tratada es que nuestra hipótesis es evolutiva, no cronológica: las edades a las que los niños acceden a un nivel formal de comprensión varian y se escalonan según la complejidad de la verbalización que se esté estudiando.

Así, en 1974 , trabajamos con 89 niños entre tres y seis años sobre la comprensión de las expresiones de limite temporal ("hasta cuándo») frente a las de límite espacial («hasta dónde»). Con respecto a los límites temporales, encontramos que la comprensión formal empieza a aparecer a 
los seis años en un 27 por 100 de los niños, pero la mayoría se distribuye, a esa edad, entre niños de comprensión espontánea (40 por 100) y niños de "transición" (33 por 100). Sin embargo, a esa misma edad (seis años), prácticamente la totalidad de los niños exhibían un modo de comprensión formal ante el "hasta» espacial (más sencillo que el temporal).

En 1977 realizamos otro trabajo sobre la comprensión de las terminaciones de género $(0, a)$ en castellano. En este caso no encontramos un tratamiento formal de la palabra hasta los trece años $(80,5$ por 100 de los niños de esta edad). En este caso los niños de "transición" tenían entre siete y nueve años.

Ahora, cuando el problema de comprensión versa sobre la distinción sujetoagente sujeto-paciente encontramos la comprensión formal a partir de los ocho años, y la "transición» en los siete años.

\section{ACLARACIONES METODOLOGICAS}

Nuestros «niveles», pues, son estrategias generales (no estadios) (Piaget et al., 1977) que el niño aplica para la comprensión del lenguaje, y cuya posibilidad de aplicación depende de múltiples factores evolutivos y madurativos.

Así, por ejemplo, una estrategia referencial para comprender la distinción sujeto agente-sujeto paciente implica no sólo la maduración semántica y sintáctica, sino también un desarrollo de la memoria tal que permita al niño mantener informaciones anteriores para poderlas comparar con las presentes.

Ias estrategias de comprensión son de orden sencuencial; por ejemplo, todos los niños que pueden comprender esta distinción de modo referencial, tienen que poderla comprender de modo espontáneo, pero no necesariamente la comprenden aún de modo formal.

Metodológicamente establecemos la correspondencia entre el orden de aparición de estas estrategias en los diferentes sujetos y la edad cronológica en tanto que escala ordinal.
La edad cronológica no funciona, pues, como variable independiente, sino que desempeña una función referencial del transcurrir del tiempo. Es decir, las variables independientes (de tipo situacional, ambiental y orgánico) provocan cambios comportamentales (estrategias de comprensión) que se miden en relación al momento en que se producen, en relación a la edad cronológica del niño.

\section{METODO}

\section{4.x. Situación y sujetos}

Hemos trabajado individualmente con 85 niños de edades comprendidas entre cinco y diez años.

Los niños han sido entrevistados personalmente por la autora, en su ambiente escolar cotidiano. Se trata de un colegio privado de clase sociocultural media-alta (padres profesionales, nivel cultural alto). Cada entrevista tuvo una duración aproximada de 30 minutos. En ella, la E. establecía un diálogo con el niño en torno a «los problemas que los niños tienen para entender a los mayores cuando hablan». La experimentadora explicaba su presencia alli como parte de un trabajo dirigido a «aprender como hablan los niños», es decir, como un trabajo de aprendizaje de la E. Se les aclaraba también que se trataba de que contestasen a las preguntas que la $\mathrm{E}$. les haría, insistiendo en que cualquier cosa que no entendiesen debían preguntarla.

La E. iba tomando nota en un protocolo del nombre y edad del niño, asi como de las contestaciones a las preguntas experimentales. Todas las contestaciones del niño iban seguidas de preguntas aclaratorias por parte de la E. del tipo "¿por qué dices eso?» o "¿qué quieres decir?» o "¿cómo lo dirías de otra manera?»"

No realizamos ninguna selección por sexos, ni utilizamos tampoco ningún criterio de selección de los niños. La E. explicaba a los profesores de los niños la naturaleza del trabajo y les rogaba que de uno en uno fuesen enviando al despacho a cualquier niño que voluntariamente qui- 
siera colaborar, sin considerar criterios clasificatorios de tipo pedagógico.

En general, tras unos diez minutos de charla "espontánea», la E. comenzaba con las preguntas del protocolo experimental.

\subsection{Procedimiento}

Prueba 1.-El diseño experimental constó de tres pruebas distintas. La primera de ellas discrimina, según la hipótesis, entre niños de nivel inferior y niños de nivel espontáneo.

Esta prueba I consistia en la comprensión de tres anécdotas contadas por la $\mathrm{E}$. Cada anécdota incluía una frase-tipo-test, cuya comprensión era necesaria para la comprensión de la anécdota total. Al final, la E. pedía a los niños que relatasen a su manera la anécdota. Los niños eran previamente advertidos de que tras escucharla se les pediría relatarla. El relato de la anécdota se acompañaba de gesticulación y entonación por parte de la $\mathrm{E}$.

\section{Anécdota I}

«Se trata de una señora que se llamaba Pepita. Pepita era un poquito fea, porque no se arreglaba y porque no se ponia vestidos bonitos. Pepita tenía una amiga que se llamaba María. Las señoras del barrio eran muy criticonas y siempre decian: "Fijate lo fea que es Pepita... Vaya pinta que tiene...", etc.

Cuando las señoras del barrio decian eso, María, su amiga, defendia a Pepita: "No tenéis razón. No es verdad. Pepita es guapa... No os metáis con ella."

Un dia estaban todas en casa de Pepita, merendando juntas. Entonces Pepita se fue al cuarto de baño y se puso muy guapa. Se pintó, se peinó, se puso un vestido bonito. Como se había marchado, las criticonas aprovecharon para decir que qué fea era. Pero entonces, de repente, entró Pepita en la habitación. Y se quedaron pasmadas, sorprendidas. Entonces su amiga María aprovecho ! dijo: "¿Lo veis? "Pepita es agradable de rer!"”"

E: "iMe has entendido...? $\lambda$ ver... cuentamelo tú ahora. Como tú te acuerdes.»

El niño hacia entonces su versión. Si en su versión no aparecía la frase-tipo-test "Pepita es agradable de ver», la F. le preguntaba explícitamente por la frase.
E.: "¿Y eso de que "Pepita es agradable de ver"? ¿Qué quiere decir?"”

Las respuestas del niño iban siendo anotadas en el protocolo.

\section{Anécdota 2}

"Un dia estaban dos amigos juntos y se estaban aburriendo. No sabían muy bien qué hacer. Entonces empezaron a pensar en buscar a otro amigo más para ver si asi se divertían: "Pues podíamos llamar a Pepe." "No, a Pepe no, que es un pesado", decia el otro. "Pues entonces podriamos llamar a Mariano." "No, a Mariano no, que se enfada siempre..." "(Oye, ¿qué te parece si llamamos a Juan?" "Si, si; llamemos a Juan. Juan es interesante de oir."

\section{Anécdota 3}

«Se trata de dos amigos que se habían ido juntos a dar un paseo. Entonces entraron en un bar a "tomarse unos vinos". Y hablaban: "¿Te acuerdas de Luisa?" "Si", dijo el otro... "Oye, le podriamos decir que se viniese con nosotros un ratito." "No, no vendrá." "Y, ¿por qué?" "Porque Luisa es enemiga de beber"."

Prueba 2.-A partir de aqui la E., explicaba al niño: "Ahora ya no te voy a contar más cuentos, y luego te pregunto una cosa y tú me contestas.»

Se introducía así la prueba 2, diseñada, según la hipótesis, para discriminar entre niños de nivel espontáneo y niños de nivel referencial.

Esta prueba constaba de seis frases seguidas de la pregunta sobre el sujeto de estas. I.a frase-tipo-test se incluía dos veces, en tercer y sexto lugar, para eliminar de los resultados el azar:

$I^{a}$ frase E.: "Las señoras hablan de lo fea que es Pepita...» "...¿Te acuerdas?" «...bueno... ¿quién habla?)”

Por este procedimiento se le planteaban al niño las seis frases.

2. frase: «Pepita se fue al cuarto de baño y se puso guapa..." "¿Quién se puso guapa?»

3." frase: "Maria dijo: Pepita es agradable de ver..." "¿Quién ve?"

4.a frase: "Maria decía que Pepita era guapa.. A "QQuién decía que Pepita era guapa?"

5." frase: "Entonces, Pepita entró en la habitación...." "¿Quién entró en la habitación?" 
6.a frase: "María dijo: Pepita es agradable de ver...» "¿Quién ve?»

Prueba 3.-Esta última prueba estaba diseñada para, según la hipótesis, discriminar entre niños de nivel referencial y niños de nivel formal.

E: «Ahora te voy a decir unas frases que no son de los cuentos..., luego te preguntare como antes... Si no entiendes, no importa, pregúntame.»

\section{Frase I}

«Ana es fácil de fastidiar...» «¿Sabes que es fastidiar?»

Si el niño decía que sí, la $\mathrm{E}$. le pedía un ejemplo, para comprobarlo. Si el niño decía que no, la E. le daba los sinónimos de «chinchar", "molestar»y "hacer rabiar». Estas aclaraciones referidas a la palabra "fastidiar» (pero no al giro "es fácil de») se daban, pues, para todos los niños.

Aclarado ésto se volvia al procedimiento experimental.

I. "Ana es fácil de fastidiar..." "¿Qué quiere decir?"

2. "Ana es fácil de fastidiar..." "¿Quién fastidia?")

La E. realizaba una pregunta sobre significado y otra sobre sujeto, repitiendo la frase antes de cada pregunta.

\section{Frase 2}

"Ana es amiga de fastidiar..."

1. "¿Qué quiere decir?" (o "¿qué es eso?»).

2. "¿Quién fastidia?"

\section{Frase 3}

«Es fácil fastidiar a Ana...»

1. "QQué quiere decir?"

2. "¿Quién fastidia?"

\section{Frase 4}

"Es amiga fastidiar a Ana...»

1. "¿Qué quiere decir?"

2. "¿Quién fastidia?"

Esta frase 4 constituye la prueba de captación del error de la E., prueba a la que hacemos amplia referencia en los resultados.

\subsection{Procedimiento de evaluación}

1. Un niño que no comprendiese, en su totalidad, las tres anécdotas, era considerado como de nivel inferior, salvo que resolviese correctamente la siguiente prueba, la de «nivel espontáneo». Ello para eliminar posibles incidencias de azar en la resolución errónea de la primera prueba.

2. Con respecto a la segunda prueba, los niños que contestaban «Pepita» en las frases $3 .^{a}$ y $6 .^{a}$ («Pepita es agradable de ver..." "¿Quién ve?") eran considerados como de nivel espontáneo, o confirmados como de nivel inferior si contestaban una vez correctamente y otra vez incorrectamente.

Los niños que contestaban cualquier otro sujeto, por ejemplo: «No sé como se llaman», o "Maria», o "sus amigas», o «las señoras", etc., eran considerados como de nivel referencial.

3. Los niños que contestaban correctamente a los tres primeras frases, los tres primeros sujetos: "Otro", "Ana", "Otro", y que captaban el error gramatical de la $4 .^{\mathrm{a}}$ frase, eran considerados como de univel formal». En cualquier otro caso, confirmábamos la pertenencia al nivel referencial.

\subsection{Cuantificación de la hipótesis}

\section{Nivel inferior:}

La Prueba I consta de tres anécdotas. Un niño de nivel inferior podría, según la hipótesis, errar las tres contestaciones. Pero por azar podría también «acertar» en una o dos de las anécdotas. Siempre que después puntuase cero en todas las demás pruebas, le considerariamos de nivel inferior. Por tanto, el esquema de actuación predicho para estos niños era:

Prueba 1.- $-1,1,2$ (ninguna, una o dos contestaciones correctas).

Prueba 2.- - (ninguna respuesta correcta). ta).

Prueba 3.- - (ninguna respuesta correc- 
Con respecto al error $\left(4 .^{a}\right.$ frase en la prueba 3) supusimos que no percibiría el error. Ello se manifestaría tanto con una mueca de incomprensión como con la adjudicación arbitraria de un significado erróneo y/o un sujeto erróneo a la fraseerror. Esta conducta está significada con un «I".

En resumen el esquema sería el explicitado en el cuadro i:

\begin{tabular}{|c|c|c|c|}
\hline Prueba I & Prueba 2 & Prueba 3 & Error \\
\hline $0,1,2$ & 0 & 0 & "I» \\
\hline
\end{tabular}

\section{Nivel espontáneo:}

En principio estos niños debían poder resolver correctamente las tres anécdotas. Así puntuarían tres en la primera prueba. No deberían poder resolver bien la segunda prueba: ante las dos frases-tipo-test ( $3 .^{\mathrm{a}}$ y $6 .^{\mathrm{a}}$ de la presentación) deberían errar ambas, o en todo caso, acertar una por azar. Por tanto, el esquema para la prueba 2 sería: 0 , 1 . Ante la prueba 3 debían fallar todas las frases $y$, por tanto, puntuar cero, aunque cabría la posibilidad de acertar una por azar, del total de tres.

Según la hipótesis, ante el Error presente en la $4{ }^{a}$ frase de la prueba 3 , estos niños deberian actuar del mismo modo que los anteriores. Conducta «I» por tanto.

Así, tal como se recoge en el cuadro 2 , el esquema de actuación predicho para estos niños sería:

\begin{tabular}{|c|c|c|c|}
\hline Prueba I & Prueba 2 & Prueba 3 & Error \\
\hline 3 & 0,1 & 0,1 & $« 1 »$ \\
\hline
\end{tabular}

Nivel referencial:

Al igual que en el caso anterior, estos niños debían resolver correctamente todas las anécdotas de la prueba ı. Por tanto, puntuarian tres. Del mismo modo, debian resolver correctamente las dos frases-tipo-test de la prueba 2 (la $3 .^{\mathrm{a}}$ y la 6. ${ }^{a}$ ). Puntuarían, por tanto: 2 . Con respecto a la prueba 3 , la frase errónea, supusimos que presentarian un nuevo tipo de conducta, llamada "2». Esta conducta debía ser incoherente. Y ello de dos modos posibles: la prueba de error contiene dos preguntas. La primera de ellas (ante «Es amiga fastidiar a Ana»), es ¿Qué significa? La segunda de ellas es ¿Quién fastidia?

Estos niños podrían decir que no significa nada, y sin embargo, después, decir que el sujeto es Ana, o podrían inventarse un significado arbitrario y luego decir que nadie fastidia porque no se entiende. Cualquiera de las posibilidades indica una captación incoherente del error que hemos denominado "conducta 2 ".

El esquema de actuación predicho para estos niños seria el del cuadro 3 :

\begin{tabular}{|c|c|c|c|}
\hline Prueba I & Prueba 2 & Prueba 3 & Error \\
\hline 3 & 2 & $0,1,2$ & «2» \\
\hline
\end{tabular}

\section{Nivel formal:}

Según la hipótesis, estos niños debían resolver correctamente la totalidad de las dos primeras pruebas (salvo un fallo en las primeras, corregido después con el acierto en todas las pruebas terceras).

Con respecto a la prueba 3 podrian fallar, por azar, una de las tres frases no-erróneas. Ello daría para esta prueba un esquema 3,4 .

Con respecto al error, la predicción para estos niños sería de una conducta de captación consistente del error: en ambas preguntas. Esta conducta la tipificamos como « 3 ». El esquema de actución sería pues:

\begin{tabular}{|c|c|c|c|}
\hline Prueba I & Prueba 2 & Prueba 3 & Error \\
\hline 3 & 2 & 3,4 & "3» \\
\hline
\end{tabular}


CUADRO $_{4}$

Cuantificación de bipótesis

\begin{tabular}{|c|c|c|c|c|}
\hline \multirow{2}{*}{$\begin{array}{c}\text { Esquema de actuación } \\
\text { predicho para }\end{array}$} & \multicolumn{3}{|c|}{ Puntuaciones } & \multirow{2}{*}{$\begin{array}{l}\text { Actuación predicha ante } \\
\text { la frase errónea (dos respuestas) }\end{array}$} \\
\hline & Prueba I & Prueba 2 & Prueba 3 & \\
\hline \multirow[t]{2}{*}{ Nivel inferior . . . . . . . } & $0,1,2$ & 0 & $\circ$ & $\begin{array}{l}\text { Adjudicación de sentido arbitraria (no percepción } \\
\text { del error). }\end{array}$ \\
\hline & & & & «1» \\
\hline \multirow[t]{2}{*}{ Nivel espontáneo ...... } & 3 & 0,1 & $0, I$ & $\begin{array}{l}\text { Adjudicación de sentido arbitraria (no percepción } \\
\text { del error). }\end{array}$ \\
\hline & & & & $\ll 1 »$ \\
\hline Nivel referencial & 3 & 2 & $0,1,2$ & $\begin{array}{l}\text { Percepción incoherente: captación del error en uno } \\
\text { de los casos o captación con explicación falsa. } \\
\text { «2» }\end{array}$ \\
\hline Nivel formal & 3 & 2 & 3,4 & $\begin{array}{l}\text { Captación consistente del error en ambas preguntas. } \\
\text { "3»" }\end{array}$ \\
\hline
\end{tabular}

\section{RESULTADOS}

El total de 85 niños, de acuerdo con la hipótesis (Cuadro 4), ha resultado distribuirse de este modo por niveles de comprensión:

\begin{tabular}{|c|c|c|}
\hline & $\begin{array}{c}\text { Núm. de } \\
\text { niños }\end{array}$ & $\begin{array}{c}\text { Edad } \\
\text { media }\end{array}$ \\
\hline Nivel inferior . . . . . . . & 8 & 5,9 \\
\hline Nivel espontáneo . . . . . . . & 18 & 6,8 \\
\hline Nivel referencial $\ldots . \ldots$. & 47 & 7,2 \\
\hline Nivel formal $: \ldots \ldots \ldots$ & 10 & 8,10 \\
\hline & $N=85$ & \\
\hline
\end{tabular}

Como deciamos en la presentación, nuestro objetivo fundamental en esta investigación eran los niños «intermedios", presuntamente «oscilatorios», y por ello entrevistamos a un número mucho mavor de niños situados entre los seis y los ocho años. Ello guiándonos por los resultados de un trabajo anterior (I ópez Ornat, 1981 ), según los cuales los niños intermedios se situaban en esas edades con respecto a esta distinción (sujeto agente-sujeto paciente).

Como resultado, el número de sujetos de nivel referencial $(\mathrm{N}=47)$ es, a nuestro entender, significativo.

No consideramos significativo el número de casos de los otros tres niveles. Consideramos que 8 , i 8 y io casos, respectivamente, son número escaso de datos para someter a prueba una hipótesis. Estaria- mos aquí de acuerdo con algunas críticas que desde dentro de la psicología cognitiva se hacen ante conclusiones establecidas sobre un número insuficiente de casos (ver Claxton 1980).

Utilizaremos, pues, los resultados de los niveles inferior, espontáneo y formal como simples referentes para una mejor matización de los resultados del nivel referencial.

\section{I. Resultados indicativos}

\section{Nivel inferior}

Los resultados indicativos del nivel inferior dan una edad media de 5,9 años. Es decir, de confirmarse esto en próximos estudios, los niños de nivel inferior presentan una conducta $(62,5$ por 100 de los casos) de no-comprensión de esta distinción, ni siquiera en condiciones de contextuación lingüística y extralingüística del problema tratado. Ante el error (75 por roo de los casos), la conducta es de no-percepción de éste. (Véase cuadro s), todo ello, por el momento, mantiene vigente la hipótesis.

\section{Nivel espontáneo}

Los resultados del nivel espontáneo dan una edad media de seis años y ocho meses, sobre un total de 18 casos. El esquema de conducta (estrategia general) 
CUADROS

Nivel inferior: puntuaciones

\begin{tabular}{|c|c|c|c|c|c|}
\hline Sujeto & Edad & Prueba : & Prueba 2 & Prueba 3 & Actuación ante el error \\
\hline 1 & 5,0 & 1 & o & $\circ$ & I \\
\hline 5 & 5,8 & 0 & 0 & 0 & I \\
\hline 9 & 5,9 & 2 & $\circ$ & $z^{*}$ & $2^{*}$ \\
\hline I I & $\int, 11$ & o & o & 0 & 1 \\
\hline 21 & 6,1 & $\mathbf{I}$ & I & $\mathrm{I}^{*}$ & $2^{*}$ \\
\hline 22 & 6,1 & 2 & 0 & 0 & I \\
\hline 23 & 6,1 & 2 & 0 & $\mathbf{1}^{*}$ & 1 \\
\hline 26 & 6,3 & $\circ$ & $\circ$ & $\circ$ & 1 \\
\hline$N=11$ & $\begin{array}{c}\text { Edad } \\
\text { media: } s, 9\end{array}$ & \multicolumn{3}{|c|}{$\begin{array}{c}37,5 \% \text { datos contradicen } \mathrm{H} \\
62,5 \% \text { datos apoyan } \mathrm{H} .\end{array}$} & $\begin{array}{c}\text { 1: } 75 \% \\
\text { 2: } 25 \% \\
3: 0 \% \\
4: 0 \% \\
25 \% \text { datos contradicen } \mathrm{H} \text {. } \\
75 \% \text { datos a favor } \mathrm{H} .\end{array}$ \\
\hline
\end{tabular}

* Con datos que contradicen hipótesis.

predicho para estos niños se confirma en el 83,4 por 100 de los casos. Es decir: la primera estrategia evolutiva de comprensión parece consistir en contextuar lingüística y extralingüísticamente, aquí y ahora, la distinción-problema para poderla comprender. Este resultado, que en esta investigación es indicativo, ha sido, sin embargo, confirmado por otros trabajos previos (López Ornat, 1974, 1975 y 1977).

Por «contextuación intralingüística» entendemos la inclusión de la frase-tipo-test en un cuento, historieta o anécdota, en una narración con sentido. Por «contextuación extralingüística» entendemos la mímica, gestos, entonación expresiva con que la narración es relatada al niño.

Con respecto a la conducta ante el error, el 55,5 por 100 de los niños actuaron según lo predicho por la hipótesis; es decir actuaton como los niños de nivel inferior. Por tanto, aunque la primera estrategia de comprensión consiste en la contextuación intra y extralingüística, en el presente, eso no permite todavia la captación de un error gramatical.

Hay, sin embargo, un 27,7 por 100 de estos niños que captaron el error inconsistentemente. Es decir, manifestaron una conducta supuestamente caracterizadora de un nivel superior (el nivel referencial). Este dato será examinado en relación a los datos obtenidos para el nivel referencial.

Véase cuadro 6 para los resultados de nivel espontáneo.

\section{Nivel formal (metalingüistico)}

Los resultados del nivel formal dan una edad media de ocho años y diez meses, sobre un total de diez sujetos. Indican que estos niños pueden comprender la distinción sujeto agente-sujeto paciente en condiciones de a-contextuación (en un 100 por 100 de los casos.)

Evidentemente la a-contextuación no es total, si se tiene en cuenta que las frases-tipo-test habian sido resueltas previamente en las dos pruebas anteriores. Pero esta situación es común a todos los niños, y sólo éstos, los mayores, pudieron resolver correctamente las cuatro frases de la $3 \cdot{ }^{\mathrm{a}}$ prueba.

La conducta de estos niños ante el error es 100 por 100 coherente con la hipótesis, es decir: perciben el error consistentemente. Sin embargo, si se observa el cuadro 4, se verá que aparece una conducta "4" no prevista en la hipótesis.

Algunos niños, un 30 por 100, captaron consistentemente el error, pero lo manifestaron diciendo "No te entiendo" ante ambas preguntas ("¿Qué significa?" 
CUADRO 6

Nivel espontáneo: puntuaciones

\begin{tabular}{|c|c|c|c|c|c|}
\hline Sujeto & Edad & Prueba $\mathrm{I}$ & Prueba 2 & Prueba 3 & Actuación ante el error \\
\hline 8 & 5,9 & 3 & 0 & 0 & $2^{*}$ \\
\hline 10 & 5,10 & 3 & ० & $\circ$ & 1 \\
\hline 14 & 6,0 & 3 & $\circ$ & $\circ$ & 1 \\
\hline I9 & 6,0 & 3 & I & I & I \\
\hline 20 & 6,0 & 3 & $\circ$ & $\circ$ & 1 \\
\hline 25 & 6,2 & 3 & $\circ$ & I & $3^{*}$ \\
\hline 28 & 6,6 & 2 & I & $2^{*}$ & I \\
\hline 30 & 6,10 & 3 & 0 & I & $3^{*}$ \\
\hline $3^{6}$ & 7,0 & 3 & 0 & I & I \\
\hline 47 & 6,9 & 3 & I & 1 & 1 \\
\hline 48 & 6,3 & 3 & 0 & $\circ$ & 1 \\
\hline 49 & 6,10 & 3 & I & I & I \\
\hline 50 & 7,0 & 3 & I & I & $2^{*}$ \\
\hline 51 & 7,2 & 3 & $\circ$ & I & $2^{*}$ \\
\hline 52 & 7,10 & 3 & o & $2^{*}$ & $3^{*}$ \\
\hline 55 & 7,9 & 3 & $\mathbf{I}$ & 2 & $2^{*}$ \\
\hline 56 & 7,10 & 3 & I & I & $2^{*}$ \\
\hline 13 & 6,6 & 3 & 1 & $2^{*}$ & I \\
\hline$N=18$ & $\begin{array}{l}\text { Edad } \\
\text { media: }\end{array}$ & \multirow{2}{*}{\multicolumn{3}{|c|}{$\begin{array}{l}83,4 \% \text { datos coherentes con } \mathrm{H} \text {. } \\
16,6 \% \text { datos no coherentes con } \mathrm{H} \text {. }\end{array}$}} & I: $55,5 \%$ Si H. \\
\hline & 6,8 & & & & $\begin{array}{c}2: 27,7 \% \\
3: 16,8 \% \mathrm{NoH} . \\
4: 0 \%\end{array}$ \\
\hline
\end{tabular}

y «¿Quién fastidia?»). Esta era la conducta "3" a la que hacíamos referencia en la hipótesis. Sin embargo, hubo un 70 por Ioo de los niños que no dijeron "No te entiendo» sino el más contundente «Asi no se habla». Es decir percibian el error y además lo achacaban a la E. Este problema se trata extensivamente en relación con los resultados del nivel referencial.

\section{CUADRO 7}

Nivel formal ("Superior»): puntuaciones

\begin{tabular}{|c|c|c|c|c|c|}
\hline 42 & 9,9 & 3 & 2 & 4 & 3 \\
\hline 43 & 10,5 & 3 & 2 & 4 & 4 \\
\hline 44 & 10,9 & 3 & 2 & 4 & 4 \\
\hline 64 & 7,9 & 3 & 2 & 3 & 3 \\
\hline 67 & 8,2 & 3 & 2 & 4 & 4 \\
\hline 73 & 8,2 & 3 & 2 & 4 & 3 \\
\hline 76 & 8,3 & . 3 & 2 & 4 & 3 \\
\hline 80 & 8,3 & 3 & 2 & 4 & 3 \\
\hline 83 & 8,4 & 3 & 2 & 4 & 3 \\
\hline 84 & 8,3 & 3 & 2 & 4 & 3 \\
\hline $\mathrm{N}=10$ & $\begin{array}{c}\text { Edad } \\
\text { media: } 8,10\end{array}$ & $100 \%$ datos & coherentes & con hipótesis. & $\begin{array}{c}\text { I: } 0 \% \\
2: 0 \% \\
3: 30 \% \\
4: 70 \% \\
\text { Datos coherentes con hipote- } \\
\text { sis (sobre la conducta } 4 \\
\text { ver explicación texto). }\end{array}$ \\
\hline
\end{tabular}




\section{Estudios}

Veamos aqui algunos problemas relacionados con el uso de la conducta ante el error para valorar la adquisición de un nivel metalingüístico (formal) de comprensión:

\subsection{Estrategia metalingüística y captación del error}

En I 961, Hill señalaba que los juicios de los sujetos (con respecto a la gramaticalidad) están afectados por demasiadas peculiaridades e idiosincrasias. Señaló también que los juicios mejoran relativamente cuando las frases no se tratan aisladas sino en relación con otras frases.

En 1965, Coleman obtuvo resultados inversos, encontrando que los hablantes nativos son capaces de organizar las frases no-gramaticales según una escala de mayor a menor gramaticalidad.

En 1969, Danks se planteó el objetivo de determinar los factores que afectan a la comprensión de las frases. Encontró que la significatividad y la gramaticalidad eran los más importantes. Siendo la significatividad el predominante.

En este sentido nuestra frase errónea («Es amiga fastidiar a Ana») es no-significativa y no-gramatical. Es decir, no tiene sentido y además no es aceptable.

Un tercer factor, el de frecuencia o cotidianeidad, se postula como variable que incide en la comprensión de las frases, y responde a la pregunta: «¿Podría darse en una conversación cotidiana?"

En 1979, Vetter, Volovecky y Howell realizaron un trabajo experimental sobre juicios de gramaticalidad contando con estos tres factores: gramaticalidad, significatividad y frecuencia, es decir, los sujetos eran preguntados sobre si las frases presentadas eran aceptables, tenian sentido y eran posibles en una conversación cotidiana.

I.os resultados indicaron que los juicios de gramaticalidad no se hacen necesariamente con independencia de los juicios de semanticidad $y$ de frecuencia. Ello debido a que, aunque los juicios eran independientes para cada factor, unos influyen en otros.

Todo ello, a nuestro entender, permite no considerar significativa la naturaleza del factor que incide en la uincomprensibilidad» de la frase, por ser estos factores interactuantes entre sí.

De hecho, como deciamos antes, nuestro error lo es en todos los sentidos: gramaticalmente, significativamente y frecuencialmente.

Vayamos, pues, a otro problema relacionado con las frases erróneas. Este se refería a la técnica experimental. Nosotros no hemos avisado a los niños de la posibilidad de que alguna frase fuese errónea.

En 1972, L. R. Gleitman, E. Shipley y H. Gleitman, en su trabajo experimental sobre juicios de gramaticalidad en niños, avisaron a éstos sobre la posible aparición de frases erróneas. Además repreguntaban al niño en el caso de que éste se equivocase.

A nuestro entender esa conducta del E., tanto como la nuestra (no avisar, no repreguntar), sesgan los resultados. De hecho, Gleitman et al., clasificaron las respuestas de los niños como correctas cuando éstas coincidian con las de los adultos. $Y$ un adulto puede juzgar la gramaticalidad (de una frase no metafórica) directamente. Gleitman et al., con su conducta experimental, eliminaron algunas variables de los resultados. Como son: la posibilidad de que el niño no haya atendido la primera vez, y la posibilidad de que el niño juzgue a priori que todo lo que el E. dice es correcto.

En nuestro trabajo, la primera variable es eliminada por el hecho de que todas las pruebas giran en torno al mismo tipo de frase; por tanto, el niño se ha habituado a esta estructura y ello elimina problemas de atención en el diseño experimental.

Con respecto al otro problema, es posible que nuestros niños hayan juzgado apriori que todo lo que el $\mathrm{E}$. dice es correcto. Como de hecho creemos que ha sucedido. Este problema es comentado extensamente en el punto sobre resultados del nivel referencial.

\subsection{Resultados del nivel referencial}

Nuestra hipótesis enuncia que el nivel referencial implica el comienzo de la con- 
ducta de distancimiento según la definen M. Lewis y S. Lee-Painter (1975). Esta conducta se concretaria en la capacidad del niño para recordar (en nuestro trabajo, la referencia dada por la E.: "Ahora te voy a decir una frase que estaba en aquel cuento de Pepita que te conté antes... ¿ ¿ Te acuerdas?") las conductas o sucesos verbales pasados (afectaría, pues, a la memoria a largo plazo).

Suponemos que ese recordar implica un mayor nivel representacional en el niño. Tal que le permite elaborar códigos efectivos de memoria. Códigos suficientemente efectivos como para permitirle aplicar una conducta de comprensión verbal previa a un problema de la misma naturaleza estructural y de distinto contenido que se presenta ahora. Pero esa misma estrategia falla cuando el niño se enfrenta con otro orden de tarea: la percepción de esa naturaleza estructural, sin contenido referencial concreto alguno. Es decir, el niño referencial todavía no comprende lo que comprende. Y aquí nos atrevemos a sugerir que esa capacidad metalingüistica podría tener que ver con una suerte de meta-memoria que estaría presente en los niños de nivel formal.

De hecho, en los estudios sobre metamemoria, centrados en el recuerdo de narraciones, aparecen varias variables determinando ese proceso.

Dos de ellas son de particular relevancia para nuestro trabajo: la estructura de la historia presentada (Mandler y Johnson, 1977; Stein y Glenn, 1979) y el contenido de la misma (Thorndyke, 1977). Ambas variables afectan asimismo a la comprensión de "Ana es fácil de fastidiar" frente a "Ana es amiga de fastidiar». El contenido de la misma ha sido mantenido como variable controlada durante nuestro experimento. (La E. se aseguró siempre de que el niño comprendía el significado de "fastidiar" equivaliéndolo a "chinchar» y «molestar», reduciendo o suprimiendo la incidencia del problema de comprensión del contenido.) Queda, pues, la comprensión de la estructura de las frases en nuestro caso. Una comprensión de lo formal.
Los niños de nivel referencial no pueden, pues, acceder a esa comprensión de lo formal, ¿por qué?

Veamos la tabla de resultados (cuadro 8):

El 66 por 100 de los niños respondieron según el esquema 3-2-O, I, 2, pero el esquema de respuesta de los datos que contradicen la hipótesis (34 por roo de los datos, 16 niños) muestra que esa contradicción se da básicamente en las respuestas a la prueba tercera. De esos 16 niños que produjeron unas respuestas contradictorias en la hipótesis, 12 de ellos lo hicieron sólo en la prueba 3.

Es decir, los niños referenciales actúan, de hecho, con un esquema 3-2-1, 2, 3 . Cuando la presentación del problema de comprensión verbal es formal, los niños de nivel referencial son desiguales en su modo de no-resolver el problema. Algunos se acercan poco $(3-2-1)$, otros mucho (3-2-3) y pocos nada $(3-2-0)$.

¿Por qué hemos incluido el esquema 3-2-3 presente en 12 de los 47 niños entre los casos de nivel referencial y no entre los casos de nivel formal? Porque incluyendo la $4^{a}$ prueba (conducta ante el error) estos niños presentan una conducta tipo «I». El esquema completo de estos I 2 niños es, pues, 3-2-3-1.

Es decir, su conducta responde al nivel formal hasta que adjudican un significado arbitrario a una frase errónea. No perciben el error, y ello lo manifiestan adjudicando un significado concreto que distorsiona la supuesta compresión estructural concretada en el esquema 3-2-3.

$\mathrm{Si}$ el niño realmente comprendiese las frases de modo metalingüístico... ¿Cómo se explica que no perciba el etror? ¿Cómo se explica que acepte una forma verbal imposible?

Tomamos, pues, la no percepción del error como condición necesaria para evaluar un nivel no-formal de comprensión.

Pero hay un 6I,8 por 100 IS I por 100 (1) $+10,8$ por $100(3)\}$ de niños de nivel referencial que manifestaron conductas contrarias a la hipótesis en cuanto a percepción del error.

Es decir, esta prueba no es predictiva de un nivel referencial: ante el error, el $5 \mathrm{I}$ 
CUADRO 8

Nivel referencial: puntuaciones

\begin{tabular}{|c|c|c|c|c|c|}
\hline Sujeto & I:dad & Prueba I & Prueba 2 & Prueba 3 & Actuación ante el crror \\
\hline 2 & 6,6 & 3 & 2 & 1 & 2 \\
\hline 3 & 5,6 & 3 & 2 & I & $\mathrm{I}^{*}$ \\
\hline 4 & 5,6 & 2 & 2 & $2^{*}$ & I* $^{*}$ \\
\hline 6 & 7,1 & 3 & 2 & 2 & $\mathrm{I}^{*}$ \\
\hline 7 & 5,9 & 1 & 2 & $\sigma^{*}$ & $1^{*}$ \\
\hline 12 & $\int, 1 \mathrm{I}$ & 2 & 2 & $1^{*}$ & $1^{*}$ \\
\hline 15 & 6,5 & 3 & 2 & 1 & $\mathrm{I}^{*}$ \\
\hline 16 & 7,0 & 3 & 2 & I & $\mathrm{r}^{*}$ \\
\hline 17 & 6,10 & 3 & 2 & o & 2 \\
\hline 18 & 6,3 & 3 & 2 & I & $\mathrm{t}^{*}$ \\
\hline 24 & 6,1 & 3 & 2 & I & $1^{*}$ \\
\hline 27 & 6,4 & 3 & 2 & 2 & 2 \\
\hline 29 & 6,8 & 3 & 2 & I & $\mathrm{I}^{*}$ \\
\hline 31 & 7,0 & 2 & 2 & $2^{*}$ & $\mathbf{1}^{*}$ \\
\hline 32 & 7,0 & 3 & 2 & 2 & $I^{*}$ \\
\hline 33 & 5,4 & 2 & 2 & I & $\mathrm{I}^{*}$ \\
\hline 34 & 6,9 & 3 & 2 & 1 & $1^{*}$ \\
\hline 35 & 6,7 & 3 & 2 & 1 & 2 \\
\hline 37 & 6,0 & 3 & 2 & o & $3^{*}$ \\
\hline 38 & 7,8 & 3 & 2 & 2 & $i^{*}$ \\
\hline 39 & 7,5 & 3 & 2 & I & $3^{*}$ \\
\hline 40 & 7,10 & 3 & 2 & $3^{*}$ & $\mathrm{i}^{*}$ \\
\hline 41 & 7,11 & 2 & 2 & 2 & 2 \\
\hline 46 & 6,11 & 3 & 2 & 2 & $\mathrm{I}^{*}$ \\
\hline 53 & 7,10 & 3 & 2 & $3^{*}$ & 2 \\
\hline 54 & 7,0 & 3 & 2 & 1 & 2 \\
\hline 57 & 8,0 & 3 & 2 & 2 & 2 \\
\hline 58 & 7,7 & 3 & 2 & 2 & 2 \\
\hline 59 & 8,1 & 3 & 2 & 2 & 2 \\
\hline 60 & $7,1 \mathrm{I}$ & 3 & 2 & 2 & 2 \\
\hline 61 & 7,9 & 3 & 2 & $3^{*}$ & 2 \\
\hline 62 & 7,9 & 3 & 2 & 2 & $3^{*}$ \\
\hline 63 & 7,7 & 3 & 2 & 2 & $3^{*}$ \\
\hline 65 & 8,6 & 3 & 2 & 1 & $1^{*}$ \\
\hline 66 & 8,3 & 3 & 2 & $3^{*}$ & $\mathrm{I}^{*}$ \\
\hline 68 & 8,0 & 3 & 2 & 2 & $I^{*}$ \\
\hline 69 & 7,7 & 3 & 2 & $3^{*}$ & 2 \\
\hline 70 & 7,7 & 3 & 2 & $3^{*}$ & $\mathrm{I}^{*}$ \\
\hline 71 & 7,6 & 3 & 2 & $3^{*}$ & 2 \\
\hline 72 & 8,1 & 3 & 2 & $3^{*}$ & $\mathrm{I}^{*}$ \\
\hline 74 & 8,2 & 3 & 2 & 2 & 2 \\
\hline 75 & $7,1 \mathbf{I}$ & 3 & 2 & $3^{*}$ & $1^{*}$ \\
\hline 77 & 8,1 & 3 & 2 & $3^{*}$ & $\mathbf{1}^{*}$ \\
\hline 78 & 7,0 & 3 & 2 & $3^{*}$ & 2 \\
\hline 79 & 8,4 & 3 & 2 & 2 & 2 \\
\hline 82 & 7,8 & 3 & 2 & 1 & $3^{*}$ \\
\hline 85 & 7,9 & 3 & 2 & $3^{*}$ & 2 \\
\hline $\mathrm{N}=47$ & $\begin{array}{l}\text { Edad } \\
\text { media: } \\
7,2\end{array}$ & \multicolumn{3}{|c|}{$\begin{array}{l}66 \% \text { datos coherentes con } \mathrm{H} . \\
34 \% \text { datos que contradicen } \mathrm{H} . \\
(\mathrm{N}=16)\end{array}$} & $\begin{array}{l}1: 51 \% \\
2: 38,2 \% \\
3: 10,8 \% \\
4: 0 \% \%\end{array}$ \\
\hline
\end{tabular}

* Datos que contradicen hipotesis. 
por roo de los niños manifestaron una conducta típica del nivel anterior (conducta del nivel espontáneo, ver cuadro 2). Un 10,8 por 100 de los niños manifestaron una conducta característica del nivel superior (aunque no fue así en las demás pruebas) y un 38,2 por 100 de los niños manifestaron la conducta esperada para el nivel referencial: percepción incoherente, captación del error ante una de las preguntas y no captación ante la otra, o captación del error "No te entiendo" seguida de una comprensión distorsionada de la frase « $i$ Ah!, iya!, ¡es que Ana fastidia a sus amigos!»: una tipica conducta oscilatoria.

Ante el error, pues, los niños del nivel referencial oscilan entre conductas "propias», conductas de un nivel inferior (mayoritariamente) y conductas de un nivel superior (minoritariamente).

Ante estos resultados, tenemos que aplicarnos, definitivamente, la crítica con la que comenzamos este trabajo. Es decir: esa conducta nítidamente oscilatoria denuncia un defecto de diseño. $O$ bien tendríamos que aceptar la validez de las conductas oscilatorias como características de estos niños intermedios, y aceptar un defecto teórico nuestro. Pero aceptarlas sólo ante el error. La mayoría de los niños del nivel referencial «descienden» de nivel ante el error ( 5 i por 100 de conductas tipo nivel inferior). Aquí cabría pensar en algo obvio que constituiría un fallo en el diseño: la percepción «incoherenten del error, tal como la hemos definido, consiste en una típica conducta oscilatoria con dos concreciones posibles. Una conducta oscilatoria entre dos conductas, una superior y otra inferior, manifestada simultáneamente. (Una conducta para cada pregunta o una conducta contradictoria; "no entender al E, pero entenderle»).

Comparemos ahora con la conducta ante el error, manifestada por los niños de niveles inferior, espontáneo y formal.

Recordamos que, por tratarse de muestras menores de 30 niños, consideramos estos datos como indicativos, no como demostrativos (ver cuadros 5, 6 y 7 ).

De ahí queda claro lo siguiente:
1. La conducta tipo i es característica del nivel inferior: 75 por 100 de los casos.

2. I a conducta tipo 2 no caracteriza a ningún nivel, sino a la diferencia entre los tres niveles y el nivel formal. Es decir, la percepción incoherente del error se da en todos los niños, menos en los de nivel formal. Aparte de eso, no discrimina entre niveles.

3. La conducta tipo 3 (captación consistente del error, como fallo del sujeto) es también caracteristica de todos los niveles menos del nivel inferior. Cero por 100 de niños de nivel inferior la manifestaron. No discrimina, pues, entre niveles, sino entre el nivel inferior y los demás.

4. La conducta tipo 4 (captación consistente del error como fallo del E.) discrimina muy claramente entre niños de nivel formal y niños de todos los demás niveles. Es específica también del nivel formal.

Por tanto, conocemos cómo es la conducta del nivel inferior y cómo la del nivel formal ante el error, pero han quedado mal definidas las conductas del nivel espontáneo y del nivel referencial ante el error. En ambos casos los niños tienden a «descender de nivel», aunque algunos manifiestan conductas incoherentes intranivel.

Pero veamos un último dato. La conducta ante el error de los niños de nivel formal (cuadro 7) ( $\mathrm{N}=10$ ).

Aparece una conducta 4 , no reseñada en nuestra presentación de la hipótesis. En los resultados observamos que algu nos niños captaban el error consistentemente, pero lo atribuían a sí mismos («no te entiendo") y, de hecho, muchos actúaban adjudicando el error a la E. (Nos parece obvio que «así no se habla» indica una adjudicación del error al experimentador, además de que éste ha sido percibido.) Esta última es la característica de la conducta predicha: percibir el error, y el cuadro de resultados agrupa tanto a los que lo percibieron y se lo adjudicaron a sí mismos, como a los que lo percibieron y se lo adjudicaron a la $\mathrm{E}$.

El criterio de agrupación de estos resultados ("3», "4") es el concepto de conflicto sociocognitivo. 
En efecto, en la última década, la escuela de Ginebra ha ido definiendo el conflicto cognitivo introduciendo el matiz "social» que se explica del modo siguiente (G. Mugny, A. N. Perret-Clermont, W. Doise, 1978) (G. Mugny, W. Doise y J. C. Deschamps, 1980): en los momentos «intermedios» del desarrollo cognitivo, en las fases de transición entre dos estadios piagetianos de desarrollo, los niños que resuelven los problemas en grupo (con otros niños de niveles superiores, con niños de niveles intermedios también, con adultos en actitud de «desconocer" la solución al problema) se benefician de la interacción, ascendiendo en su nivel evolutivo. Ello se observa a través de un paradigma de pretest-interacción-postest.

Este dato, y otros trabajados con el mismo paradigma, apoyan la tesis de que el conflicto cognitivo que define a los niños «intermedios» es en realidad un conflicto "sociocognitivo" en donde el esquema cognitivo diferente de otro sujeto que interactúa con el sujeto intermedio se explicita, se manifiesta, èn la relación entre los niños (o los niños y los adultos) al intentar resolver desde posiciones distintas el mismo problema.

$\mathrm{Si}$ el niño «intermedio» establece una relación social, accede a la posibilidad de conocer el esquema cognitivo que ahora sólo intuye.

Así podríamos suponer que el problema error que nosotros le hemos planteado a los niños ha creado un conflicto cognitivo. No a los niños de niveles inferior ni espontáneo (sin prerrequisito cognitivo), sino a los niños que pueden tener ese conflicto (nivel referencial y nivel formal).

Creemos que al plantearle un error a un niño, sin preaviso de que esto se puede producir, el aspecto social del conflicto puede determinar los resultados, nublando las caracteristicas cognitivas de la conducta.

Esto es lo que parece haber sucedido con la conducta ante el error en niños referenciales: los datos son muy poco nitidos. Esta hipótesis se sustenta no sólo sobre esa apariencia confusa de los datos, sino también sobre los resultados del nivel formal. Hemos visto cómo algunos niños (30 por 100) parecian "no atreverse» a suponer que la $\mathrm{E}$. pudiera «hablar mal».

Este factor puede haber incidido con mucha más fuerza en los niños referenciales, puesto que su estrategia de comprensión del lenguaje es aún imperfecta (necesitan recurrir al recuerdo de situaciones concretas previas) y, de hecho, manifiestan ante el error cualquier tipo de conducta (ver cuadro 3).

Podriamos, quizá con la estrategia experimental ya mencionada de L. Gleitman, haber reducido la incidencia de ese factor social en el conflicto, avisando al niño previamente de que «el E. puede equivocarse»".

En este caso, nos hubiera quedacio la duda sobre cuál es el comportamiento adulto ante el mismo problema. Esto, pues, define la naturaleza de nuestro próximo trabajo: el mismo problema planteado a adultos, también sin previo aviso sobre el error. Quizá los adultos no necesiten preaviso para manifestar una conducta de tipo "4": el error es del experimentador; de hecho, téngase en cuenta que esta conducta fue manifestada por el 70 por 100 de los niños de nivel formal. Por tanto, es esperable en adultos en un mayor porcentaje. De todos modos, los resultados del nivel formal, como decíamos antes, son sólo indicativos, por estar obtenidos sobre io sujetos.

Un próximo trabajo intentará elucidar, pues, el problema de las pruebas de captación de errores como modos válidos de evaluar la adquisición de una estrategia metalingüística (nivel formal) de comprensión del lenguaje hablado.

\section{CONCLUSIONES}

Hemos realizado una prueba de ji cuadrado para descartar la hipótesis nula. Situando los datos en una matriz con los cuatro niveles evolutivos y cuatro grupos de edad: niños de entre 5,6 y 6,5 años de edad, niños entre 6,6 y 7,5 , niños de 7,6 y $8,5 \mathrm{y}$, por último, niños mayores de 8,6 años. 
El valor de $\chi^{2}$ es de 47,4648 , que con nueve grados de libertad resulta ser significativo con $\mathrm{p}<0,001$.

Puesto que la probabilidad de azar es menor del I por 1.000 , ello nos permite afirmar que la distribución de niños en los niveles hipotetizados se debe a las estrategias de comprensión del lenguaje que hemos agrupado por niveles.

Tomando la forma metalingüística de compresión del lenguaje como la forma más evolucionada de comprensión, los resultados de ésta y anteriores investigaciones permiten afirmar que el proceso de adquisición de esa forma metalingüistica de compresión pasa por cuatro "niveles», que son cuatro conjuntos de conductas de comprensión que se diferencian entre si por el modo de relación que el niño necesite establecer con el contexto de verbalización para entenderla. Asi:

I. Nivel inferior: encontrado para esta distinción (sujeto agente - sujeto paciente) hasta los seis años. En este nivel no se da comprensión de la verbalización estudiada, aunque ésta se produzca en condiciones de máxima contextuación: intra y extralingüistica, presente y simultánea a la verbalización.

2. Nivel espontáneo: encontrado, para esta distinción, entre los seis y los siete años. Este nivel se define por una conducta de comprensión de la verbalización cuando (y sólo cuando) ésta se produce contextuada tanto lingüística como extralingüísticamente (incluida en un cuento y, además, relatada con lenguaje gestual).

3. Nivel referencial: objeto central de esta investigación, se encuentra entre los siete y los ocho años. La conducta de comprensión se da aunque la verbalización se presente aislada de ambos contextos. Sin embargo, el niño recurre a establecer una relación entre la verbalización y un contexto concreto pero interno (referencia), operación que se apoya sobre el recuerdo de contextos anteriores. Si esta posibilidad de referencia (recurrir a concretar lo abstracto) le es impedida, el niño pierde la posibilidad de comprender la verbalización.

4. Nivel formal o metalingüistico: encontrado para esta distinción a partir de los ocho años. La conducta de comprensión de la verbalización se independiza de toda necesidad de contextuación interna o externa, y el niño presenta la habilidad para comprender la verbalización directamente, apoyado como los adultos, en las claves intralingüísticas y en el contexto de la emisión.

\section{Referencias}

BNIJFs, P. B.; RfFse, H. W., y Nessfirondi, J. R. Métodos de investigación en psicologia evolutiva: enfogue del ciclo vital. Madrid: Morata, $198 \mathrm{I}$.

CI.ARK, E. C. Non-linguistic Strategies and the acquisition of word meanings. Cognition, 1973, 2 (2), I 61-182.

Ci.AXTon, G. Cognitive psychology: a suitable case for what sort of treatment? En Claxton G. C. (Fid.): Cognitive Psycbology, new directions. Londres: Routledge and Kegan Paul, i980.

Col.EMAN, E. B. Responses to a scale of grammaticalness. Journal of Verbal Learning and Verbal Bebavior. $1965,4,521-527$.

Cromer, R. Desarrollo del lenguaje y del conocimiento. En Foss, B. M. (Ed.): Nuetas perspectivas en el desarrollo del niño. Madrid: Fundamentos, 1978.

DinkS, J.H. Grammaticalness and Meaningfulness in the Comprehension of Sentences. Journal of $\mathrm{l} / \mathrm{erbal}$ Learning and Verbal Bebavior. 1969, 8, 687-696.

Dolse W.; Deschamps, J. C., y MLigni, G. Psicología social experimental. Barcelona: Hispano-Europea, 1980.

Gi.eitMin, L. R. Maturational Determinants of Language Growth. Cognition. 1981, I, 104-114.

Gifitmin, I. R.; Shipi.ey, E., y Gi.fitmin, H. The emergence of child as grammarian. Cognition. 1972, $1,137-164$.

His., A. A. Grammaticality. Word, 1961, 17, 1-10.

Lewis, M., y LeF-PAINTER, S. The origins of interactions: methodological issues. Fn Riegel, K. F. y Rosenwald, G. C. Structure and Transformation. Nueva York: Wiley, 1975.

López ORnat, S. Concepto y estructura lingüística en la dinámica de una adquisición verbal. Tesis doctoral. Publicada en extracto. Madrid, 1974.

- Interpretación de estructuras superficiales: el género. Trabajo no publicado. Madrid, 1975 (a).

- Relaciones lenguaje-pensamiento. Revista de Psicología general y aplicada. 1975 (b), 137.

- Dinámica de la adquisición verbal. Revista de Psicologia general y aplicada. 1975 (c), vol. 30, 137, 29-34. 
- Un ovejo tiene cinco patas. Lenguaje: interpretaciones evolutivas de la señal. Revista de Psicologia general y aplicada. 1977, 145, $211-224$.

- Interacción e internalización en la evolución cognitiva: proyecto. Revista de clinica y análisis grupal. 1981, 26.

Mindier, J., y JoHnSon, N. S. Remembrance of things parsed: story structure and recall. Cognitive Psychology. 1977, 9, 1 I-191.

MARCHESI, A. El desarrollo del conocimiento sobre los propios procesos cognitivos. En Actas del VII Congreso Nacional de Psicologia. Santiago de Compostela, 1982.

Mugny, G.; Perret-Ci.ermont, A. N., y Doise, W. Coordinaciones interpersonales y diferencias sociológicas en la construcción del intelecto. Revista de Clinica y análisis grupal. $1978,12$.

OI.ERON, P. Langage et developpement mental. Bruselas: Dessart, 1972.

Ortony, A.; SChAl.iert, D. L.; Reynol.dS, R. E., y ANTOS, S. J. Interpreting Metaphors and Idioms: some effects of context on Comprehension. Journal of Verbal Learning and Verbal Bebavior. 1978, $17,465-477$.

PingeT, J. La toma de conciencia. Madrid: Morata, 1976.

- et al.: Los estadios en la psicología del niño. Buenos aires: Nueva Visión, 1977.

- et al.: Mesa redonda sobre las tesis de J. Piaget. Revista de Clinica y Análisis grupal. 1980, 21.

REYNol.dS, R. E., y ORTONY, A. Some issues in the measurement of children's comprehension of metaphorical language. Child Development. 1980, 51, 1.1 10-1.1 19.

STEIN, N. L., y Gi.ENN, C. G. An analysis of story comprehension in elementary school children. En Freedle, R. O. (Ed.): Discurse processing: multidisciplinary perspectives. Norwood: N. J. Ablex, 1979.

ThoRndike, P. W. Cognitive structure in comprehension and memory of narrative discourse. Cognitive Psycbology. 1977, 9, 7-110.

VETTER, H. J.; VoloveCKY, J., y How'El.l., R. Judgements of grammaticalness: a partial replication and extension. Jowrnal of Psycholinguistic Research. 1979, 8, núm. 6.

Vil.1.IERS, P. A., y ViJ.l.IERS, J. G. Primer lenguaje. Madrid: Morata, 1980.

WEIR, R. H. Language in the crib. La Haya: Mouton, 1970 (I.a edición, 1962).

WeXI.ER, K., y CUI.ICover, P. W. Formal principles of language acquisition. Cambridge, Mass.: MIT Press, 1980.

WII.ENSKI, R. Meta-planning: representing and using knowledge about planning in problem solving and natural language understanding. Cognitive Science. 1981, , 197-233. 